\title{
Map Model for Nonlinear Alpha Particle Interaction with Toroidal Alfvén Waves
}

\author{
H. L. Berk, B. N. Breizman, ${ }^{\text {a) }}$ and Huanchun Ye \\ Institute for Fusion Studies \\ The University of Texas at Autin \\ Austin, Texas 78712
}

DOE/ET/53088--566

DE93 001851

\begin{abstract}
A map model has been developed for studying the nonlinear interaction of alpha particles with the toroidal Alfven eigenmodes. The map is constructed by assuming a linear interaction during a single poloidal transit, which allows the study of the nonlinear interaction over many transits. By using this map, analytic expressions are obtained for the particle nonlinear bounce frequency, and the wave amplitude threshold for the onset of particle orbit stochasticity. The map model can also facilitate self-consistent simulations which incorporate the time variation of the waves.
\end{abstract}

PACS No. 52.35.Qz, 52.20.Dq, 52.65.+z, 51.10.+y

a) Permanent address: Institute of Nuclear Physics, Novosibirsk 630090, Russia

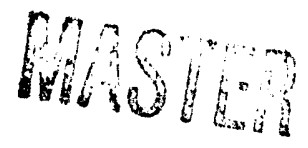




\section{INTRODUCTION}

This paper is motivated by the problem of alpha particle confinement in a tokamak under thermonuclear conditions. We address the question of how the alpha particles are affected by the shear Alfven waves which can be unstable due to the spatial gradient of the alpha particle distribution. As a particular example, we consider the the excitation of the toroidicity-induced Alfven eigenmodes (TAE) which has been the topic of interest in many recent studies. ${ }^{1-5}$

An essential question about alpha particle confinement is whether one can have the wave saturate at a level below the threshold of global particle stochastic diffusion. If the saturation level is below the threshold, then only a small portion of the alpha particles that are in the resonance region has significant interactions with the wave, and these particles remain in a limited region of phase space. Hence the instability is relatively benign, and particle losses are small. On the other hand, if the saturation level exceeds the stochasticity threshold, then the particles are no longer confined in a limited phase space region, and the overall free energy of the particle distribution is available to drive the wave amplitude to even higher levels, which in turn enhances particle losses. In this paper we basically concentrate on the determination of the threshold for the onset of particle stochastic diffusion due to overlapping of neighboring resonances. By comparing this threshold with the previously found saturation amplitude for a single unstable mode,${ }^{6,7}$ we formulate the conditions under which the losses of alpha particles, due to the excitation of TAE mode, are guaranteed to be very low. Much higher losses can be expected when the stochasticity threshold is exceeded. In order to understand whether these enhanced losses are still acceptable for a reactor, one needs a self-consistent simulation of the wave-particle interaction in the stochastic regime. The approach developed in this paper may considerably simplify such simulations.

The stochasticity threshold can be determined by studying the test particle motion in a given wave field. A similar approach has been used for studying the effects of ripples ${ }^{8,9}$ 
and low-frequency perturbations. ${ }^{10} \mathrm{~A}$ procedure that allows for an analytic estimate, or rapid numerical calculation, is the reduction of the particle motion to a two-dimensional map. As the wave amplitudes are sufficiently small, for a single transit the response of alpha particles can be calculated using linear theory with the linear structure of the TAE mode. The nonlinear dynamics of the alpha particles, the simplest form! of which is the oscillation at a nonlinear bounce frequency $\omega_{b}$, is then simulated by following the map for many transits.

The reduction to a map is made possible by a number of preliminary simplifications based on the physical nature of the problem. In our problem, we consider only the passing particles in a large aspect ratio tokamak. Most of these particles are far enough from the

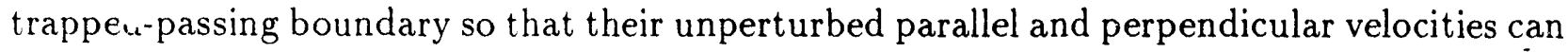
be treated as constant. We also use the fact that it is the toroidal angular momentum, rather than the particle energy, that primarily changes during the alpha particle interaction with TAE modes. Despite these simplifications, many important physical details are retained by the map. For example, the structure of the resonant interaction for a given particle depends on where the unperturbed orbit is located relative to the radial structure of the mode, and on the ratio of the orbit width to the mode width. Of particular interest is the case when the particle excursion (due to the guiding center drift) from the flux surface is comparable or even larger than the radial width of the mode structure. For example, in the linear theory ${ }^{11}$ that has been developed, which is closely related to the map model presented here, it was shown that the instability drive is substantially reduced compared to what would be extrapolated using the thin-orbit theory. Along with the changes in the growth rate, new resonances appear when the orbit is thick; these resonances being negligible for the thin orbit case. It will also be shown that there is an important range of parameters where the alpha particle response is insensitive to the finite Larmor radius (FLR) effects, even if the scale length of the radial mode structure is comparabie or less than the Larmor radius. This fact 
allows for an essential analytic simplification for describing the particle-wave interaction in both the linear theory ard the mapping method.

With all these elements included, the structure of the map is still sufficiently tractable that analytic estimates for $\omega_{b}$ and the stochasticity threshold can be made. Several numerical tests presented in Sec. 4 illustrate the accuracy of the analytic estimates.

The mapping technique allows for efficient computer simulation of nonlinear alpha particle dynamics, by allowing the time step size to be a poloidal transit time. Using an area-preserving map, we can follow a particle in low amplitude fields for a long time and guarantee that the diffusion of a particle is not due to the inaccuracy of the approximate equations.

The rest of the paper is organized as follows. In Sec. II we derive the map equations. Then in Sec. III we use the map to obtain analytic expressions for the nonlinear bounce frequency $\omega_{b}$, and the threshold amplitude for the onset of orbit stochasticity. In Sec. IV we present some of the numerical results for the map. In Sec. V we discuss how to change the wave amplitude self-consistently, and how to make a simulation code based on the mapping technique. Finally in Sec. VI we summarize the results. Appendix A contains a detailed derivation of a form factor which is used in Sec. II. It also includes the finite Larmor radius correction neglected in the main text.

\section{DERIVATION OF MAP}

In our derivation we shall restrict ourselves to the low $\beta$ limit, in which case both the perturbed electric and the magnetic fields, $\delta \mathbf{E}$ and $\delta \mathbf{B}$, have negligible parallel components. This allows a perturbed field representation of the form

$$
\delta \mathbf{E}=-\nabla \delta \phi-\frac{1}{c} \partial_{t} \delta A_{\|} \mathbf{b}, \quad \delta \mathbf{B}=\nabla \times\left(\mathbf{b} \delta A_{\|}\right) \doteqdot-\mathbf{b} \times \nabla \delta A_{\|}
$$


with

$$
\partial_{t} \delta A_{\|}=-c \mathbf{b} \cdot \nabla \delta \phi
$$

where $\mathbf{b} \equiv \mathbf{B} / B$ is the direction of local equilibrium magnetic field. For a single mo ie with frequency $\omega$, we have $\delta A_{\|}=(c / i \omega) \mathbf{b} \cdot \nabla \delta \phi$.

To describe the particle motion, we siart with the phase-space Lagrangian

$$
L=\dot{\mathbf{r}} \cdot\left[M \mathbf{v}+\frac{e}{c} \mathbf{A}(\mathbf{r})+\frac{e}{c} \mathbf{b} \delta A_{\|}(\mathbf{r}, t)\right]-\left[\frac{1}{2} M v^{2}+e \delta \phi(\mathbf{r}, t)\right],
$$

where $e$ and $M$ are the alpha particle charge and mass, respectively, and $\mathbf{r}$ and $\mathbf{v}$ are independent variables. $\mathbf{A}(\mathbf{r})$ is a vector potential for the equilibrium magnetic field: $\nabla \times \mathbf{A}=\mathbf{B}$. We model the tokamak in the large aspect ratio limit assuming the flux surfaces to be circular. Then the equilibrium magnetic field is $\mathbf{B}=B_{0}\left[1 \cdots\left(r / R_{0}\right) \cos \theta\right] \hat{\boldsymbol{\phi}}+B_{\theta}(r) \hat{\boldsymbol{\theta}}$, where $r$ is the flux surface radius, $R_{0}$ is the major radius of the magnetic axis, $\theta$ is the poloidal angle, $\hat{\theta}$ and $\hat{\phi}$ are the poloidal and toroidal unit vectors, and $B_{\theta}(r)=r B_{0} / R_{0} q(r)$, with $q$ the safety factor. The vector potential $\mathbf{A}$ can then be expressed in terms of the toroidal and poloidal flux functions, $\chi$ and $\psi$, by

$$
\mathbf{A}=\chi(r) \nabla \theta-\psi(r) \nabla \varphi
$$

where

$$
\chi \doteqdot \frac{1}{2} B_{0} r^{2}, \quad \psi \doteqdot B_{0} \int_{0}^{r} \frac{r^{\prime} d r^{\prime}}{q\left(r^{\prime}\right)}
$$

and $d \psi / d \chi=1 / q$.

The unperturbed orbit of a particle is determined by three invariants: magnetic moment $\mu \equiv M v_{\perp}^{2} / 2 B$, energy $E \equiv M v^{2} / 2$, and the toroidal angular momentum $p_{\varphi} \equiv R M v_{\varphi}-$ $(e / c) \psi$, where $R=R_{0}+\dot{r} \cos \theta$. The corresponding unperturbed trajectory in the $(r, \theta)$ plane is implicitly determined by the relation

$$
p_{\varphi}=-\frac{e}{c} \psi(r) \pm R \sqrt{2 M(E-\mu B)}
$$


where the \pm signs correspond to moving parallel or anti-parallel to $\mathbf{b}$, respectively. For particles far from the trapping-passing boundary, which are of primary interest in our inrestigation, $v_{\|}$and $v_{\perp}$ can be treated as constant. Then Eq. (6) sirnplifies to (here and after we neglect, in the unperturbed motion, the distinction between the particle and the guiding-center )

$$
r=\bar{r}+\Delta_{b} \cos \theta
$$

where $\bar{r}$ is the average distance of a particle from the magnetic axis, defined by

$$
p_{\varphi}=-\frac{\epsilon}{c} \psi(\bar{r}) \pm R_{0} \sqrt{2 M\left(E-\mu B_{0}\right)}
$$

and $\Delta_{b}$, the particle orbit excursion from the flux surface $r=\bar{r}$, is given by

$$
\Delta_{b} \equiv \frac{\left(v_{\|}^{2}+\frac{1}{2} v_{\perp}^{2}\right) q(\bar{r})}{v_{\|} \Omega}
$$

with $\Omega \equiv \epsilon B_{0} / M c$ the alpha particle gyrofrequency. Although using expression (7) requires the orbit excursion from the flux surface, $\Delta_{b}$, to be small compared to the minor radius, $r$, this excursion can still be larger than the characteristic width of TAE modes, which is roughly of the order of $r^{2} / n q R_{0}$, with $n$ the toroidal mode number. ${ }^{12}$

The statement that variations in $v_{\|}$and $v_{\perp}$ are negligible is also valid when a perturbation is present. In order to justify this we first show that relative change in $\bar{r}$ produced by the TAE mode is much larger than that produced in the particle energy. The perturbed quantities in a single TAE mode depend on time, $t$, and toroidal angle, $\varphi$, as $\exp i(n \varphi-\omega t)$. As $t$ and $p$ enter the Lagrangian $L$ only through this particular combination, then $L$ is invariant with respect to transformation

$$
t \mapsto t+\tau, \quad \varphi \mapsto \varphi+\frac{\omega}{n} \tau .
$$

Thus, although neither $p_{\rho}$ nor $E$ is conserved in presence of perturbation, their following combination is still invariant:

$$
E-\frac{\omega}{n} p_{\varphi}=\text { const }
$$


The change in the particle energy is then related to the change in $\bar{r}$ by $\Delta E \doteqdot(\omega e / n c) \psi^{\prime} \Delta \bar{r}$. Thus we obtain the following estimate

$$
\frac{\Delta v}{v} \doteqdot \frac{\omega}{\omega_{* \alpha}} \frac{\Delta \bar{r}}{L_{\alpha}}
$$

where $L_{\alpha}$ is the alpha particle density scale length, and

$$
\omega_{* \alpha} \equiv \frac{n q \rho_{\alpha} v}{2 \bar{r} L_{\alpha}}
$$

is the alpha particle diamagnetic frequency ( $\rho_{\alpha}$ denotes the alpha particle gyroradius). It is known from previous studies ${ }^{13}$ that $\omega<\omega_{* \alpha}$ is required for TAE modes to be unstable. To simplify our analysis we shall assume 2 stronger inequality $\omega \ll \omega_{* \alpha}$, which is certainly well satisfied for moderate values of $n$. Thus we can neglect the relative change in particle energy compared to the change in the mean radius.

To proceed with our derivation, it is convenient to make a change of variable

$$
\mathbf{u}=\mathbf{v}+\frac{e}{M c} \mathbf{b} \delta A_{\|}
$$

Note that since $\delta A_{\|}$remains small in realistic situations, $\mathbf{u}$ will remain close to $\mathbf{v}$ for all time, so for all practical purposes we can ignore the difference between them. This simple transformation removes the perturbation from the coefficient of $\dot{\mathbf{r}}$ in Eq. (3) and simplifies the subsequent calculations:

$$
L=\left[M \mathbf{u}+\frac{e}{c} \mathbf{A}(\mathbf{r})\right] \cdot \dot{\mathbf{r}}-\frac{1}{2} M u^{2}-e \sum_{p}\left[\left(1-\frac{u_{\|} \mathbf{b}}{i \omega} \cdot \nabla\right) \delta \phi_{p}(\mathbf{r}, t)+c . c .\right],
$$

where $p$ is a mode index. For each mode we have eliminated $\delta A_{\|}$in terms of $\delta \phi$ and dropped the term quadratic in $\delta \phi$. Note that the first term in $L$ now contains only the unperturbed fields, so we can perform the usual guiding-center transformation, and obtain the guidingcenter Lagrangian. ${ }^{14} \mathrm{As}$ the gyroradius is assumed to be small compared to the equilibrium 
scale $\mathrm{k} \cdot \mathrm{gth}$, we need only keep the gyroradius in the perturbed potential $\delta \phi_{m}$. Therefore the gyroaveraged Lagrangian becomes

$$
\begin{aligned}
\bar{L}=[ & \left.M u_{\|} \mathbf{b}+\frac{e}{c} \mathbf{A}\right] \cdot \dot{\mathbf{X}}-\left[\frac{1}{2} M u_{\|}^{2}+\mu B_{0}\left(1-\frac{r}{R_{0}} \cos \theta\right)\right. \\
& \left.+e \sum_{p}\left(1-\frac{u_{\|} \mathbf{b}}{i \omega} \cdot \nabla\right)\left\langle\delta \phi_{p}\right\rangle+\text { c.c. }\right],
\end{aligned}
$$

where $\mathbf{X}$ is the guiding-center position, $\mu \doteqdot M u_{\perp}^{2} / 2 B_{0}$ is the magnetic moment, and the angular bracket stands for gyroaverage. It turns out that the finite Larmor radius (FLR) is less important for moderate $n$ values compared to the finite drift orbit effects, so for our purpose we shall henceforth neglect in the main text the FLR corrections. A heuristic argument for neglecting the FLR effects has been given in Ref. 11. The appropriate modification with FLR correction is given in Appendix A. FLR effects for the case of large mode numbers have been studied in Ref. 15 .

Now, substituting Eq. (4) into the guiding-center Lagrangian gives

$$
\bar{L}=p_{\theta} \dot{\theta}+p_{\varphi} \dot{\varphi}-\left[\frac{1}{2} M u_{\|}^{2}+\mu B_{0}\left(1-\frac{r}{R_{0}} \cos \theta\right)+e \sum_{p}\left(1-\frac{u_{\|} \mathbf{b}}{i \omega} \cdot \nabla\right) \delta \phi_{p}+c . c .\right],
$$

where

$$
p_{\theta} \equiv \frac{e}{c} \chi(r)+M u_{\|} r \frac{B_{\theta}}{B} \doteqdot \frac{e}{c} \chi(r)
$$

and $p_{\varphi}$ now denotes the guiding-center toroidal angular momentum

$$
p_{\varphi} \equiv-\frac{e}{c} \psi(r)+M u_{\|} R=-\frac{e}{c} \psi(r)+M u_{\|}\left(R_{0}+r \cos \theta\right) .
$$

To a very good approximation the toroidal motion of passing particles can be described by $\varphi \doteqdot u_{\|} t / R_{0}+\varphi_{0}$. Using this, the Lagrangian reduces to one for a single degree of freedom

$$
L=p_{\theta} \dot{\theta}-\left\{-\frac{u_{\|}}{R_{0}} p_{\varphi}+\frac{1}{2} M u_{\|}^{2}+\mu B_{0}\left(1-\frac{r}{R_{0}} \cos \theta\right)+e \sum_{p}\left[\left(1-\frac{u_{\|} \mathbf{b}}{i \omega} \cdot \nabla\right) \delta \phi_{p}+\text { c.c. }\right]\right\} .
$$


The second term is the Hamiltonian for the reduced motion. Using Eq. (17) for $p_{\varphi}$ and discarding all terms that are independent of $\theta$ and $r$, we obtain the "effective" Hamiltonian

$$
H\left(p_{\theta}, \theta ; t\right)=\frac{e u_{\|}}{c R_{0}} \psi(r)-\frac{e}{c} B_{0} v_{d} r \cos \theta+e \sum_{p}\left[\left(1-\frac{u_{\|} \mathbf{b}}{i \omega} \cdot \nabla\right) \delta \phi_{p}+c . c .\right],
$$

where $r$ is a function of $p_{\theta}$ through Eq. (16), and $v_{d} \equiv\left(u_{\|}^{2}+\frac{1}{2} u_{\perp}^{2}\right) / \Omega R_{0}$.

The equations of motion can then be obtained easily by

$$
\dot{p}_{\theta}=-\frac{\partial H}{\partial \theta}, \quad \dot{\theta}=\frac{\partial H}{\partial p_{\theta}} .
$$

However, for the map model it is more convenient to use $\theta$ as the independent variable instead of $t$. In the Hamiltonian formulation it is very easy to make this conversion, by noting that the action can be written in two ways:

$$
S=\int d t\left[p_{\theta} \dot{\theta}-H\right]=-\int d \theta\left[H \frac{d t}{d \theta}-p_{\theta}\right]
$$

By comparison of the forms, we see that the new canonical variables are $(H, t)$ and the new Hamiltonian is $p_{\theta}$. The mean radius, $\bar{r}$, is now defined as a function of $H$ by

$$
H \equiv \frac{e u_{\|}}{c R_{0}} \psi(\bar{r})
$$

Expanding $\psi$ about $\bar{r}$, we can solve Eq. (19) to obtain

$$
p_{\theta}(H, t ; \theta)=\frac{e}{c} \chi(\bar{r})+\frac{e}{c} B_{0} \bar{r} \Delta_{b} \cos \theta-\frac{e}{\omega_{t}} \sum_{p}\left[\left(1-\frac{u_{\|} \mathbf{b}}{i \omega} \cdot \nabla\right) \delta \phi_{p}+c . c .\right]
$$

where $\Delta_{b} \equiv v_{d} / \omega_{t}$ is the orbit excursion length, $\omega_{t} \equiv u_{\|} / R_{0} q(\bar{r})$ is the poloidal transit frequency. The unperturbed part of Eq. (23) gives the unperturbed guiding center orbit (7).

The equations of motion in terms of $(H, t)$ are now given by

$$
\frac{d H}{d \theta}=-\frac{\partial p_{\theta}}{\partial t}, \quad \frac{d t}{d \theta}=\frac{\partial p_{\theta}}{\partial H} .
$$

In the absence of perturbation we have, neglecting the variation of $\Delta_{b}$,

$$
\left(\frac{d}{d \theta}\right)_{0} H=0, \quad\left(\frac{d}{d \theta}\right)_{0} t=\frac{1}{\omega_{t}} .
$$


To treat the perturbed part we substitute Eq. (7) into

$$
\delta \phi_{p}(r, \theta, \varphi, t) \equiv \sum_{m} \phi_{m}(r) e^{i(n \varphi-m \theta-\omega t)}
$$

and Fourier expand in $\theta$, to obtain

$$
\delta \phi_{p}=\sum_{m} \phi_{m}\left(\bar{r}+\Delta_{b} \cos \theta\right) e^{i(n \varphi-m \theta-\omega t)}=\frac{1}{2} \sum_{m, \ell} \phi_{m, \ell} e^{-i[(m+\ell) \theta+\bar{\omega} t]},
$$

with $\bar{\omega} \equiv \omega-n u_{\|} / R_{0}, \mathbf{b} \cdot \nabla=i k_{\|} \equiv i R_{0}^{-1}(n-m / q)$, and the Fourier amplitudes $\phi_{m, \ell}$ defined by

$$
\phi_{m, \ell} \equiv \int_{0}^{2 \pi} \frac{d \theta}{\pi} \phi_{m}\left(\bar{r}+\Delta_{b} \cos \theta\right) e^{i \ell \theta}
$$

Now integrating $d H / d \theta$ along the unperturbed orbit for one transit between $-\pi$ and $\pi$, taking care so that $t$ always increases, we obtain the change of $H$ due to the perturbation

$$
H_{N+1}-H_{N}=-\frac{\epsilon}{\left|\omega_{t}\right|} \sum_{m, \ell}\left(1-\frac{k_{\|} u_{\|}}{\omega}\right) i \bar{\omega} \frac{\sin \pi \Psi_{m+\ell}^{\prime}}{\Psi_{m+\ell}^{\prime}} \phi_{m, \ell} e^{-i \bar{\omega} t_{N}}+c . c .,
$$

where $\Psi_{j}^{\prime} \equiv j+\bar{\omega} / \omega_{t}$ is the rate of phase change alon $\mathrm{g}$ the unperturbed orbit. Notice that as $\omega-k_{\|} u_{\|}=\omega_{t} \Psi_{m}^{\prime}$, we can simplify the above equation to

$$
H_{N+1}-H_{N}=\operatorname{sgn}\left(u_{\|}\right) \frac{e B_{0}}{c} \sum_{p} \frac{i \bar{\omega}}{\omega} K\left(\bar{r}_{N+1}\right) e^{-i \bar{\omega} t_{N}}+c . c .,
$$

where

$$
K \equiv \frac{c}{B_{0}} \sum_{m, \ell} \frac{\sin \pi \Psi_{m+\ell}^{\prime}}{\Psi_{m+\ell}^{\prime}} \ell \phi_{m, \ell}
$$

and we have neglected terms that vanish at resonance (which occurs where $\Psi_{m+\ell}^{\prime} \doteqdot 0$ or $\left.\Psi_{m}^{\prime} \doteqdot-\ell\right)$.

The change of $t$ can in principle be calculated by integrating $d t / d \theta$. Alternatively, we determine the change in $t$ by ensuring that $\left(t_{N}, H_{N}\right) \mapsto\left(t_{N+1}, H_{N+1}\right)$ is a canonical 
transformation. Using the standard technique we introduce a mixed-variable generating function $F\left(t_{N}, H_{N+1}\right)$, such that

$$
H_{N}=\frac{\partial F}{\partial t_{N}}, \quad t_{N+1}=\frac{\partial F}{\partial H_{N+1}}
$$

The appropriate generating function is found to be

$$
F\left(t_{N}, H_{N+1}\right)=t_{N} H_{N+1}+\operatorname{sgn}\left(u_{\|}\right) \frac{e}{c}\left\{2 \pi \chi\left(\bar{r}_{N+1}\right)-\sum_{p} \frac{B_{0}}{\omega}\left[K\left(\bar{r}_{N+1}\right) e^{-i \bar{\omega} t_{N}}+c . c .\right]\right\}
$$

Substituting it into Eq. (32) we finally obtain the following implicit map

$$
\begin{aligned}
& H_{N+1}=H_{N}+\operatorname{sgn}\left(u_{\|}\right) \frac{e B_{0}}{c} \sum_{p}\left(\frac{i \bar{\omega}}{\omega} K\left(\bar{r}_{N+1}\right) e^{-i \bar{\omega} t_{N}}+c . c .\right) \\
& t_{N+1}=t_{N}+\frac{1}{\left|\omega_{t}\left(q_{N+1}\right)\right|}\left[2 \pi-\left(\frac{2}{\omega} \frac{\partial K}{\partial r^{2}}\left(\bar{r}_{N+1}\right) e^{-i \bar{\omega} t_{N}}+c . c .\right)\right],
\end{aligned}
$$

where $\bar{r}_{N+1} \equiv \bar{r}\left(H_{N+1}\right)$, and $q_{N+1} \equiv q\left(\bar{r}_{N+1}\right)$.

To apply Eqs. (34) to particular TAE modes one first has to find $\phi_{m, \ell}$ by calculating the integral (28) for a given mode structure. The integral can either be done numerically or evaluated analytically when a simple representation for the mode structure is available. To proceed analytically we will restrict ourselves to the case in which $\phi_{m, \ell}$ is determined by a single gap. In the vicinity of the gap location $r_{m}$, where $q\left(r_{m}\right)=\left(m+\frac{1}{2}\right) / n$, the radial structure of TAE modes has the following generic form $\mathrm{m}^{12}$

$$
\phi_{m}(r)=\Phi_{m} \int_{r_{m}}^{r} \frac{\Delta_{m}+\alpha_{m}\left(r^{\prime}-r_{m}\right)}{\left(r^{\prime}-r_{m}\right)^{2}+\Delta_{m}^{2}} d r^{\prime}
$$

where $\Delta_{m}$ is the "local mode width." From the linear theory of TAE modes" $\Delta_{m} \approx r_{m}^{2} / m s R_{0}$ with $s \equiv r q^{\prime} / q$. In addtion to the above local structure the mode also has a global structure, with a scale length $L_{m} \approx r_{m} / m s$, neglected in Eq. (35). This simplification requires the particle excursion from the flux surface to be much less than $L_{m}$, which sets a limit on the maximum mode number that can be rigorously treated. Note that the map model itself is more general than expression (35). We merely use Eq. (35) to illustrate 
the apabilities of the model. The result can then be improved by using the actual mode structure determined numerically. The calculations of the Fourier amplitudes $\phi_{m, \ell}$ for the mode structure (35) are given in Appendix A, where we find the result for $\ell \neq 0$ to be

$$
\phi_{m, \ell}=-\frac{\Phi_{m}}{|\ell|}\left[i\left(z^{|\ell|}-\text { c.c. }\right)+\alpha_{m}\left(z^{|\ell|}+\text { c.c. }\right)\right] \text {, }
$$

where $z \equiv-(x+i y)+\left[(x+i y)^{2}-1\right]^{1 / 2}, x \equiv\left(\bar{r}-r_{m}\right) / \Delta_{b}, y \equiv \Delta_{m} / \Delta_{b}$, and the branch of the square root is chosen so that $|z|<1$.

In the next section we simplify Eqs. (34) about the center of an isolated resonance, and calculate the nonlinear bounce frequency $\omega_{b}$ and the stochasticity threshold. Then in Sec. 4 we shall discuss the numerical implementation of Eq. (34).

\section{NONLINEAR BOUNCE FREQUENCY AND RESONANCE OVERLAPPING CONDITION}

Under the influence of a single finite amplitude wave, particles oscillate spatially. The amplitude of oscillation is largest if the particle is in a resonance region (labeled by $\ell_{0}$ ) where $\Psi_{m+\ell_{0}}^{\prime} \approx 0$, so that $\sin \left(\pi \Psi_{m+\ell}^{\prime}\right) / \Psi_{m+\ell}^{\prime} \approx \pi \delta_{\ell \ell_{0}}$. This leads to a reduced map wherefrom analytic expressiuns for the separatrix width and the characteristic nonlinear bounce frequency can be obtained straightforwardly. In the rest of this section we omit the subscript 0 . The validity of this procedure requires the width of resonance to be smaller than the distance between the adjacent resonances. It is well known ${ }^{16}$ that by equating the resonance width to the distance between adjacent resonances, a good estimate for the onset of stochasticity can be obtained.

The resonance condition $\Psi_{m+\ell}^{\prime}=0$ defines the radial position of an island

$$
q\left(r_{*}\right)=\frac{m+\ell}{n} \frac{1}{1-\omega R / n u_{\|}} \approx \frac{m+\ell}{n}\left(1+\frac{\omega R}{n u_{\|}}\right) .
$$


Expanding the map about the center of the island by letting $r_{N} \equiv r_{*}+\delta r_{N}$ and $t_{N} \equiv$ $2 \pi . N / \omega_{t}\left(r_{\star}\right)+\tau_{N}$, we obtain

$$
\begin{aligned}
\delta r_{N+1}-\delta r_{N} & =\frac{2 \pi}{\left|\omega_{t}\right|} \frac{\bar{\omega}}{\bar{\omega}} \frac{c}{B_{0} r_{*}}\left|\ell \phi_{m, \ell}\right| \sin \left(\bar{\omega} \tau_{N}-\beta_{m, \ell}\right)=\frac{\partial \delta r}{\partial N}, \\
\tau_{N+1}-\tau_{N} & =\frac{2 \pi}{\left|\omega_{t}\right|} \frac{q^{\prime}}{q} \delta r_{N+1} \doteqdot \frac{\partial \tau}{\partial N},
\end{aligned}
$$

where $\ell \phi_{m, \ell}=\left|\ell \phi_{m, \ell}\right| e^{i \beta_{m, \ell}}$, and $\omega_{T}$ etc. are evaluated at $r_{*}$. These equations describe the oscillation of particles trapped in the island. Combining Eqs. (38) gives the equation for a nonlinear pendulum:

$$
\frac{\partial^{2} \tau}{\partial N^{2}}=\left(\frac{2 \pi}{\omega_{t}}\right)^{2} \frac{\bar{\omega}}{\omega} \frac{s c}{B_{0} r_{*}^{2}}\left|\ell \phi_{m, \ell}\right| \sin \left(\bar{\omega} \tau-\beta_{m, \ell}\right)
$$

where $s \equiv r q^{\prime} / q$ is the local magnetic shear. Without loss of generality we can put $\beta_{m, \ell}=\pi$. For a particle deeply trapped inside the island, $\sin (\bar{\omega} \tau) \approx \bar{\omega} \tau$, so that we immediately see that the number of steps, $N_{b}$, that is needed for a particle to return to its original position (one bounce) is given by

$$
N_{b}=\left|\frac{\omega_{t}}{\bar{\omega}}\right|\left[\frac{s c}{\omega r_{*}^{2} B_{0}}\left|\ell \phi_{m, \ell}\right|\right]^{-1 / 2} .
$$

Denoting the trapping frequency by $\omega_{b}$, we have

$$
\omega_{b} \equiv N_{b}^{-1} \omega_{t}=|\bar{\omega}|\left[\frac{s c}{\omega r_{*}^{2} B_{0}}\left|\ell \phi_{m, \ell}\right|\right]^{1 / 2} .
$$

To obtain the separatrix width associated with Eq. (38), we construct, in a standard manner, the first integral of Eq. (38). Dividing the first equation by the second we obtain .

$$
\delta r^{2}-\frac{4 c}{\omega s B_{0}}\left|\ell \phi_{m, \ell}\right| \cos ^{2}\left(\frac{\bar{\omega} \tau}{2}\right)=C
$$

The sign of the constant $C$ determines whether a particle is trapped by the wave or not. The separatrix occurs when $C=0$, with the separatrix equation given by

$$
\delta r^{ \pm}= \pm\left[\frac{4 c}{\omega s B_{0}}\left|\ell \phi_{m, \ell}\right|\right]^{1 / 2} \cos \left(\frac{\bar{\omega} \tau}{2}\right) .
$$


The maximum width of the separatrix is therefore

$$
\Delta r_{s}=4\left[\frac{c}{\omega s B_{0}}\left|\ell \phi_{m, \ell}\right|\right]^{1 / 2}=\frac{4 r_{*}}{s} \frac{\omega_{b}}{|\bar{\omega}|} .
$$

We note that our derivation assumed $\Delta r_{s} \partial \ln \left|\ell \phi_{m, \ell}\right| / \partial r<1$.

To investigate the resonance overlap we observe that the resonance condition (37) gives the distance between neighboring resonances

$$
\left(r_{*}\right)_{\ell+1}-\left(r_{*}\right)_{\ell} \approx \frac{1}{n q^{\prime}} .
$$

Therefore the resonance overlapping criterion $\Delta r_{s} \geq\left(r_{*}\right)_{\ell+1}-\left(r_{*}\right)_{\ell}$ yields the threshold amplitude for the onset of particle orbit stochasticity

$$
\omega_{b} \geq \frac{|\bar{\omega}|}{i n q} .
$$

To get a feeling for this threshold in terms of physical quantities, we use Eq. (36) for a particle that crosses the mode surface, and has the orbit width $\Delta_{b}$ much larger than the local mode width $\Delta_{m}$. For such a particles, $|z| \approx 1$, which yields $\ell \phi_{m, \ell} \sim 2 \Phi_{m} \sim\left(2 r v_{A} / m c\right) \delta B_{r}$. Thus we obtain, with $m \approx n q$ and $\omega \approx v_{A} / 2 q R_{0}$,

$$
\frac{\delta B_{r}}{B_{0}} \geq \frac{r_{*}}{64 m R_{0}} \frac{1}{q s} \text {. }
$$

For typical tokamak parameters, it gives the threshold $\delta B_{r} / B_{0} \approx 1.5 \times 10^{-3} / \mathrm{m}$. This result is in qualitative agreement with the earlier numerical integration results obtained by Sigmar et al. $^{17}$

To conclude this section, we note that in arriving at the stochasticity threshold (47) we used Eq. (45), which is valid only for a single pair of $(n, m)$ (i.e., a single gap). In the presence of many modes, the distance between resonances can be reduced (roughly by a factor $1 / N$ with $N$ the number of modes), which results in a lower stochasticity threshold (reported recently in Ref. 18). This situation will be illustrated by some of the numerical results in the next section. 


\section{NUMERICAL RESULTS}

In the numerical implementation of map (34) we choose Eq. (35) with $\alpha_{m}=0$ for the mode structure, and 'ise expressions (31) and (36). We solve the first of Eqs. (34) for $\bar{r}_{N+1}\left(\bar{r}_{N}, t_{N}\right)$ by Newton's method, then evaluate $t_{N+1}$ directly using the second equation. The symplectic structure of the map ensures that particles can be followed accurately for many poloidal transits without introducing nonphysical secular behavior arising from the approximations in the equations.

The parameters that we choose are as follows:

$$
\frac{a}{R_{0}}=0.25, \quad q(r)=1+2\left(\frac{r}{a}\right)^{2}, \quad \frac{v_{A}}{2 a \Omega}=0.03,
$$

where $a$ is the minor radius of the plasma column. In the following figures we use $q$ as the radial variable, with $q=1$ corresponding to $r=0$ and $q=3$ corresponding to $r=a$. The wave amplitude is normalized as

$$
A_{m} \equiv \frac{m c}{r v_{A} B_{0}} \Phi_{m} \approx \frac{\delta B_{r}}{B_{0}} .
$$

Figures 1-3 are for the case of a single mode, with mode numbers $n=3, m=6$. They are surface-of-section plots for seven particles, all having $v=v_{\|}=v_{A}$, but are initially located at different radii with $q=1.67,1.83,2.0,2.17,2.33,2.5,2.67$, respectively. The vertical axis is $q(\vec{r})$, while the horizontal axis, the phase, is defined as $\bar{\omega} t \bmod 2 \pi$. Each orbit is mapped for 2000 poloidal transits.

Figure 1 has the wave amplitude $A_{m}=6.25 \times 10^{-5}$, which is equal to $1 / 4$ of the estimated stochasticity threshold as given by Eq. (47) in the previous section. The three islands correspond to the principal (the middle one) and the two nearest sideband resonances. The thin stochastic layer around the principal resonance appears because the orbit is close to the separatrix. We can also see that away from the resonances the orbits are perturbed very little. 
Figure 2 has the wave amplitude $A_{m}=1.25 \times 10^{-4}$, which is $1 / 2$ of the estimated stochastic threshold. The principal and the lower sideband resonances have overlaped, and the three orbits in the overlapping region become indistinguishable.

Figure 3 has the wave amplitude $A_{m}=2.5 \times 10^{-4}$, which is equal to the estimated threshold value. The stochasticity has almost spreadout to the whole resonance region. Note, however, that the stochastic region is still bounded by invariant curves, and that the particle transport is thus confined.

For global particle transport to occur, one need either higher wave amplitudes (for larger islands), or the presence of many modes (for closer islands). Figure 4 shows the radial locations of resonances for different mode numbers, according to Eq. (37). Only the principal resonance (indicated by solid lines) and two neighbouring sidebands (indicated by dashed lines) are included. The numbers atop the solid lines are the $m$-values, while the $n$-values are shown at the bottom. At the right we stack all the resonances together to show their tendency to form clusters. Within each cluster stochasticity can occur at very low wave amplitudes. Notice that for these mode numbers there still exists a relatively wide radial interval, labeled by $A$, which is without any resonances. This region may prevent a particle in the inner region from being transported to the outside.

Figure 5 is the surface-of-section plot for two particles in presence of 12 modes, with $n=3, m=4-8$ and $n=4, m=5-11$. All modes have the amplitude $A_{m}=2.5 \times 10^{-5}$, which is $1 / 10$ the estimated threshold value. One particle is initially located at $q=2.17$, which is above region $A$. This particle can clearly diffuse to the plasma edge. The other particle is created at $q=1.62$, which is below region $A$. We see that this particle is confined due the the absence of resonances in region $A$. Both particles are mapped for 2000 poloidal transits. For the inner particle to diffuse to the outside, it is necessary to have higher wave amplitudes, or to have some modes whose resonances are inside region $A$. In Figure 7 we 
raise the wave amplitudes to $A_{m}=5.55 \times 10^{-5}$. In this case the inner particle can overcome the barrier presented by region $A$, and diffuse to the plasma edge.

\section{CHANGE OF PARTICLE ENERGY}

The map model also allows for an efficient calculation of the energy transfered from a particle to a wave as the former completes one turn. Within the guiding center approximation we have

$$
(\Delta E)_{N}=-e \int_{t_{N}}^{t_{N+1}} d t \mathbf{v}_{d} \cdot \delta \mathbf{E},
$$

where $\mathbf{v}_{d}=-v_{d l}(\hat{\mathbf{r}} \sin \theta+\hat{\boldsymbol{\theta}} \cos \theta)$ is the guiding center drift velocity, and $\delta \mathbf{E}$ is the perturbed electric field. Neglecting the relatively unimportant contribution from $\delta E_{\theta}$, we can then carry out the integration along the unperturbed orbit, and obtain

$$
\begin{aligned}
(\Delta E)_{N} & =-\sum_{p} e \int_{-\pi}^{\pi} \frac{d \theta}{|\dot{\theta}|} v_{d} \sin \theta \frac{\partial \phi_{p}}{\partial r} e^{-i[m \theta+\bar{\omega} t(\theta)]}+c . c . \\
& =\operatorname{sgn}\left(u_{\|}\right) \frac{e B_{0}}{c} \sum_{p} K\left(\bar{r}_{N}\right) i e^{-i \bar{\omega} t_{N}}+c . c .
\end{aligned}
$$

where $K$ is given by Eq. (31). Notice the similarity between Eqs. (51) and (30). Aside from a normalizing constant, the only significant difference is the factor $\omega / \bar{\omega}$. This factor can be explained by noting that $H$ is essentially equal to $E-p_{\varphi} u_{\|} / R_{0}$, the particle energy in the rotating frame, and that $p_{\varphi}$ is related to $E$ by Eq. (11). Since in computing the map we have already found the value of $K$, it is straightforward to calculate $\Delta E$. As the above expression is a sum over modes, it is natural to interpret each term in the sum to be the energy gained by the respective wave. Such a model is in the spirit of quasilinear theory.

A self-consistent simulation scheme utilizing the map may be constructed as following. We first choose a time step $T$, which is long compared to the particle transit time but short compared to the wave growth time. Each particle is then mapped for many turns until the total time just exceeds $T$, and its energy change is accumulated. The particle energy at 
time $T$ can then be found by an appropriate interpolation method. Next we use Eq. (51) to calculate the energy transfered to each wave and thus update its amplitude self-consistently according to

$$
W E_{i+1}=\left(1-\gamma_{d} T\right) W E_{i}+\sum \Delta E
$$

where $\gamma_{d}$ is damping rate of TAE mode due to the background plasma, and the sum means accumulation of $\delta E$ for the duration $T$ and over all particles.

\section{CONCLUSIONS}

In conclusion, we have developed a map model for describing the nonlinear interaction between the alpha particles and toroidal Alfven waves, in particular the TAE modes. Using this model we have obtained analytic expressions for the nonlinear bounce frequency and the critical wave amplitude for the onset of particle orbit stochasticity, both are essential quantities for assessing the alpha particle confinement in a fusion tokamak.

Using the stochasticity threshold obtained in Sec. 3 and the estimates for the saturation level of a single TAE mode driven by alpha particles, we can now formulate the condition under which the effect of the mode on alpha particle losses is expected to be insignificant. This must be the case when the mode saturates below the stochastic threshold. The nonlinear analysis presented in Refs. 6,7 shows that the value of $\omega_{b}$ at saturation is the largest of the two quantities: the linear growth rate $\gamma_{L}$ and $\gamma_{L}\left[\left(\nu / \gamma_{d}\right)\left(\omega / \gamma_{L}\right)^{2}\right]^{1 / 3}$, where $\gamma_{d}$ is the background dissipation rate for the TAE mode, $\nu$ is the $90^{\circ}$ pitch angle scattering rate for the alpha particles, and $\omega$ is the mode frequency. By combining these estimates with Eq. (46) we conclude that stochastic diffusion is negligible when

$$
\frac{\bar{\omega}}{4 n q}>\max \left\{\gamma_{L} ; \gamma_{L}\left[\frac{\nu}{\gamma_{d}}\left(\frac{\omega}{\gamma_{L}}\right)^{2}\right]^{1 / 3}\right\} \text {. }
$$


Note that this criterion is only good for rough scaling estimates whereas exact numerical factors in it have to be found from numerical simulations. It should also be noted that violation of Eq. (53) does not necessarily lead to global stochasticity. it may result in particle diffusion within only the width of the banana orbit. Global diffusion requires either the presence of many modes with different radial positions over the poloidal cross section, or the mode numbers to be sufficiently high so that the mode would have substantial components near different gaps being crossed by the particle orbit. The regime of global diffusion is of course unfavorable for the confinement of the alpha particles. However, even this regime may still be acceptable if one does not go too far beyond the instability threshold. To get a better feeling about global diffusion one has to study the corresponding quasilinear problem with the source of alpha particles and background damping mechanism for the TAE modes taken into account.

\section{ACKNOWLEDGMENTS}

We would like to thank D. J. Sigmar and C. T. Hsu for many stimulating discussions. We would also like to thank P. J. Morrison, D. del Castillo-Negrete, and C. Kueney for numerous discussions on symplectic maps. This work was supported by the US Department of Energy Contract No. DE-FG05-80ET-53088. 


\section{APPENDIX A. CALCULATION OF FORM FACTUR}

In this appendix we present the detailed calculation of the Fourier coefficient $\phi_{m, \ell}$, as defined by

$$
\phi_{m, \ell} \equiv \int_{0}^{2 \pi} \frac{d \theta}{\pi} \phi_{m}\left(\bar{r}+\Delta_{b} \cos \theta\right) e^{i \ell \theta}
$$

with the radial made profile given ky Eq. (35). Clearly $\phi_{m, \ell}=\phi_{m,-\ell}$, so we shall consider only the case $\ell \geq 0$. Differentiate both sides of Eq. (A.1) with respect to $\bar{r}$ we have

$$
\begin{aligned}
\frac{\partial \phi_{m, \ell}}{\partial \bar{r}} & =\int_{0}^{2 \pi} \frac{d \theta}{\pi} \frac{\partial \phi_{m}}{\partial r}\left(\bar{r}+\Delta_{b} \cos \theta\right) \stackrel{?}{i \ell \theta}^{i \ell \theta} \\
& =\frac{\Phi_{m}}{\pi} \int_{0}^{2 \pi} d \theta \frac{\Delta_{m}+\alpha_{m}\left(\bar{r}-r_{m}+\Delta_{b} \cos \theta\right)}{\left(\bar{r}-r_{m}+\Delta_{b} \cos \theta\right)^{2}+\Delta_{m}^{2}} e^{i \ell \theta}
\end{aligned}
$$

Defining

$$
x \equiv \frac{\bar{r}-r_{m}}{\Delta_{b}}, \quad y \equiv \frac{\Delta_{m}}{\Delta_{b}}
$$

we can rewrite Eq. (A.2) as

$$
\frac{\partial \phi_{m, \ell}}{\partial x}=\frac{\Phi_{m}}{\pi} \int_{0}^{2 \pi} d \theta \frac{y+\alpha_{m}(x+\cos \theta)}{(x+\cos \theta)^{2}+y^{2}} e^{i \ell \theta} .
$$

This integral can be evaluated by countour integration method. Let $\zeta \equiv e^{i \theta}$, then $d \theta=d \zeta / i \zeta$, $\cos \theta=\left(\zeta+\zeta^{-1}\right) / 2$. Substituting into the above equation we obtain

$$
\begin{aligned}
\frac{\partial \phi_{m, \ell}}{\partial x} & =\frac{\Phi_{m}}{\pi i} \oint_{|\zeta|=1} d \zeta \zeta^{\ell-1} \frac{y+\alpha_{m}\left[x+\frac{1}{2}\left(\zeta+\zeta^{-1}\right)\right]}{\left[x+i y+\frac{1}{2}\left(\zeta+\zeta^{-1}\right)\right]\left[x-i y+\frac{1}{2}\left(\zeta+\zeta^{-1}\right)\right]} \\
& =\frac{\Phi_{m}}{\pi i} \oint_{|\zeta|=1} d \zeta \zeta^{\ell}\left[\frac{\alpha_{m}+i}{\zeta^{2}+2(x+i y) \zeta+1}+\frac{\alpha_{m}-i}{\zeta^{2}+2(x-i y) \zeta+1}\right] \\
& =\frac{\Phi_{m}}{\pi i} \oint_{|\zeta|=1} d \zeta \zeta^{\ell}\left[\frac{\alpha_{m}+i}{\left(\zeta-\zeta_{1}\right)\left(\zeta-\zeta_{2}\right)}+\frac{\alpha_{m}-i}{\left(\zeta-\zeta_{3}\right)\left(\zeta-\zeta_{4}\right)}\right]
\end{aligned}
$$

For real $y$, the four poles are related to each other by $\zeta_{2}=1 / \zeta_{1}, \zeta_{3}=\zeta_{1}^{*}, \zeta_{4}=1 / \zeta_{1}^{*}$, where star denotes complex conjugate. For definiteness, let $\zeta_{1}=z$, where

$$
z \equiv-(x+i y)+\sqrt{(x+i y)^{2}-1}, \quad|z|<1
$$


so that $\zeta_{1}$ is inside the unit circle. Now deform the countour by shrinking it to the origin, we pick up the pole contributions from two poles $\zeta_{1}$ and $\zeta_{3}$, yielding

$$
\frac{\partial \phi_{m, \ell}}{\partial x}=2 \Phi_{m}\left[\frac{\left(\alpha_{m}+i\right) z^{\ell}}{z-z^{-1}}+\left(\frac{\left(\alpha_{m}^{*}+i\right) z^{\ell}}{z-z^{-1}}\right)^{*}\right] \text {. }
$$

On the other hand from Eq. (A.6) we have

$$
z+\frac{1}{z}=-2(x+i y)
$$

Differentiate both sides with respect to $x$ we obtain

$$
z-\frac{1}{z}=-\frac{2 z}{\partial z / \partial x}
$$

Substituting it into Eq. (A.7) we obtain

$$
\frac{\partial \phi_{m, \ell}}{\partial x}=-\Phi_{m}\left[\left(\alpha_{m}+i\right) z^{\ell-1} \frac{\partial z}{\partial x}+\left(\left(\alpha_{m}^{*}+i\right) z^{\ell-1} \frac{\partial z}{\partial x}\right)^{*}\right]
$$

The right-hand side has become a differential. For $\ell>0$ we have

$$
\phi_{m, \ell}=-\frac{\Phi_{m}}{\ell}\left[\left(\alpha_{m}+i\right) z^{\ell}+\left(\alpha_{m}-i\right) z^{* \ell}\right]+\text { const }
$$

The constant of integration can be shown to vanish by taking the limit $|x| \rightarrow \infty$. Therefore we have finally

$$
\ell \phi_{m, \ell}=-\Phi_{m}\left[\alpha_{m}\left(z^{\ell}+\text { c.c. }\right)+i\left(z^{\ell}-\text { c.c. }\right)\right]
$$

For reference, we also display result for $\ell=0$ :

$$
\phi_{m, 0}=-\Phi_{m}\left[\left(\alpha_{m}+i\right) \ln z+\left(\alpha_{m}-i\right) \ln z^{*}\right]+\text { const. }
$$

although this result is not used in this paper.

Expression (A.12) becomes especially simple when $\Delta_{b}$ is much larger than $\Delta_{m}$. In this limiting case one can put $\Delta_{m}=0$ and rewrite Eq. (A.12) in the form

$$
\ell \phi_{m, \ell}=2 \Phi_{m}\left[\sin \ell \theta_{0}-\alpha_{m} \cos \ell \theta_{0}\right]
$$


where

$$
\cos \theta_{0} \equiv \frac{r_{m}-\bar{r}}{\Delta_{b}} \quad\left(0 \leq \theta_{0} \leq \pi\right)
$$

We now generalize this result to the case of finite Larmor radius $\rho$. This only requires $\bar{r}$ to be replaced by $\vec{r}-\rho \sin \alpha$ with subsequently averaging Eq. (A.14) over the gyrophase $\alpha$. Thus Eqs. (A.14) and (A.15) transform to

$$
\begin{gathered}
\ell \phi_{m, \ell}=2 \Phi_{m}\left\langle\sin \ell \theta_{\rho}-\alpha_{m} \cos \ell \theta_{\rho}\right\rangle, \\
\cos \theta_{\rho} \equiv \frac{r_{m}-\bar{r}-\rho \sin \alpha}{\Delta_{b}}
\end{gathered}
$$

where the angular bracket means gyroaveraging according to

$$
\langle f(\alpha)\rangle=\int_{0}^{2 \pi} \frac{d \alpha}{2 \pi} f(\alpha) .
$$

Assuming $\rho$ to be much less than $\Delta_{b}$ and $\theta_{0}$ not too close to 0 or $\pi$ we rewrite Eq. (A.17) as

$$
\theta_{\rho}=\theta_{0}+\frac{\rho \sin \alpha}{\Delta_{b} \sin \theta_{0}}
$$

With this expression for $\theta_{\rho}$ gyroaveraging gives

$$
\ell \phi_{m, \ell}=2 \Phi_{m}\left[\sin \ell \theta_{0}-\alpha_{m} \cos \ell \theta_{0}\right] J_{0}\left(\ell \rho / \Delta_{b} \sin \theta_{0}\right)
$$

where $J_{0}$ is the zeroth order Bessel function. Thus if $\ell \rho / \Delta_{b}$ is less than 1 , there is no essential change in the function $\phi_{m, \ell}$. 


\section{REFERENCES}

1 K. L. Wong, Phys. Rev. Lett. 66, 1874 (1991).

2 W. W. Heidbrink, E. J. Strait, E. Doyle, and R. Snider, Nucl. Fusion 31, 1635 (1991).

3 C. Z. Cheng and M. S. Chance, Phys. Fluids 29, 3695 (1986).

${ }^{4}$ F. Zonca and L. Chen, Phys. Rev. Lett. 68, 592 (1992).

${ }^{5}$ M. N. Rosenbluth, H. L. Berk, J. W. Van Dam, and D. M. Lindberg, Phys. Rev. Lett. 68, $596(1992)$.

${ }^{6}$ H. L. Berk, B. N. Breizman, and H. Ye, Phys. Rev. Lett. 68, 3563 (1992).

7 H. L. Berk and B. N. Breizman, Phys. Fluids B 2, 2246 (1990).

8 R. J. Goldston, R. B. White, and A. H. Boozer, Phys. Rev. Lett. 47, 647 (1981).

9 S. V. Konovalev and S. V. Putvinskii, Fusion Technology 18, 397 (1990); S. V. Putvinskii, "Alpha Particles in a Tokamak," Voptosy Teorii Plasmy (Reviews of Plasma Physics) 18, Edited by B. B. Kadomtsev (Moscow, 1990, in Russian).

${ }^{10}$ H. E. Mynick, "Transport of Energetic Ions by Low-n Magnetic Fluctuations," submitted to Phys. Fluids B (1992).

11 H. L. Berk, B. N. Breizman, and H. Ye, Phys. Lett. A 162, 475 (1992).

12 M. N. Rosenbluth, H. L. Berk, J. W. Van Dam, and D. M. Lindberg, Phys. Fluids B 4, 2189 (1992).

13 G. Y. Fu and J. W. Van Dam, Phys. Fluids B 1, 1949 (1989).

14 R. G. Littlejohn, J. Plasma Phys. 29, 111 (1983).

15 G. Y. Fu and C. Z. Cheng, accepted for publication in Phys. Fluids B (1992).

${ }^{16}$ B. V. Chirikov, Phys. Reports 52, 265 (1979).

${ }^{17}$ D. J. Sigmar, C. T. Hsu, R. White, and C. Z. Cheng, Phys. Fluids B 4, 1506 (1992).

${ }^{18}$ C. T. Hsu, D. J. Sigmar, and D. Spong, "Alpha Physics in Tokamaks," presented at The Third Workshop on Alpha Physics in TFTR (MIT, Cambridge, May 1992). 


\section{FIGURE CAPTIONS}

1. Surface of section plot with seven orbits for the case of a single mode. The mode amplitude is $1 / 4$ of the estimated threshold value for the onset of orbit stochasticity. See Sec. IV for details.

2. Similar to Fig. 1, except that the mode amplitude is $1 / 2$ of the estimated threshold value for the onset of orbit stochasticity.

3. Similar to Figs. 1 and 2, except that the mode amplitude is equal to the estimated threshold value for the onset of orbit stochasticity.

4. The radial locations of the resonances (principal plus two neighboring sidebands) for $n=1$ to 4 . Notice that the resonances tend to form clusters, and that in this case there is a significant radial interval (region $A$ ) that is withot resonances. This region may act as a barrier to particle transport.

5. Surface of section plot for two particles in presence of twelve modes. The mode amplitude is $1 / 10$ of the estimated threshold value given in Sec. III. One particle is born above region $A$ and it can reach the plasma edge within 2000 poloidal transits. The other particle is born below region $A$. Notice that this region limits the outward particle transport. See Sec. IV for more details.

6. Surface of section plot for the inner particle of Fig. 5, but with higher wave amplitudes. Notices that in this case the particle has overcome the transport barrier presented by region $A$. 


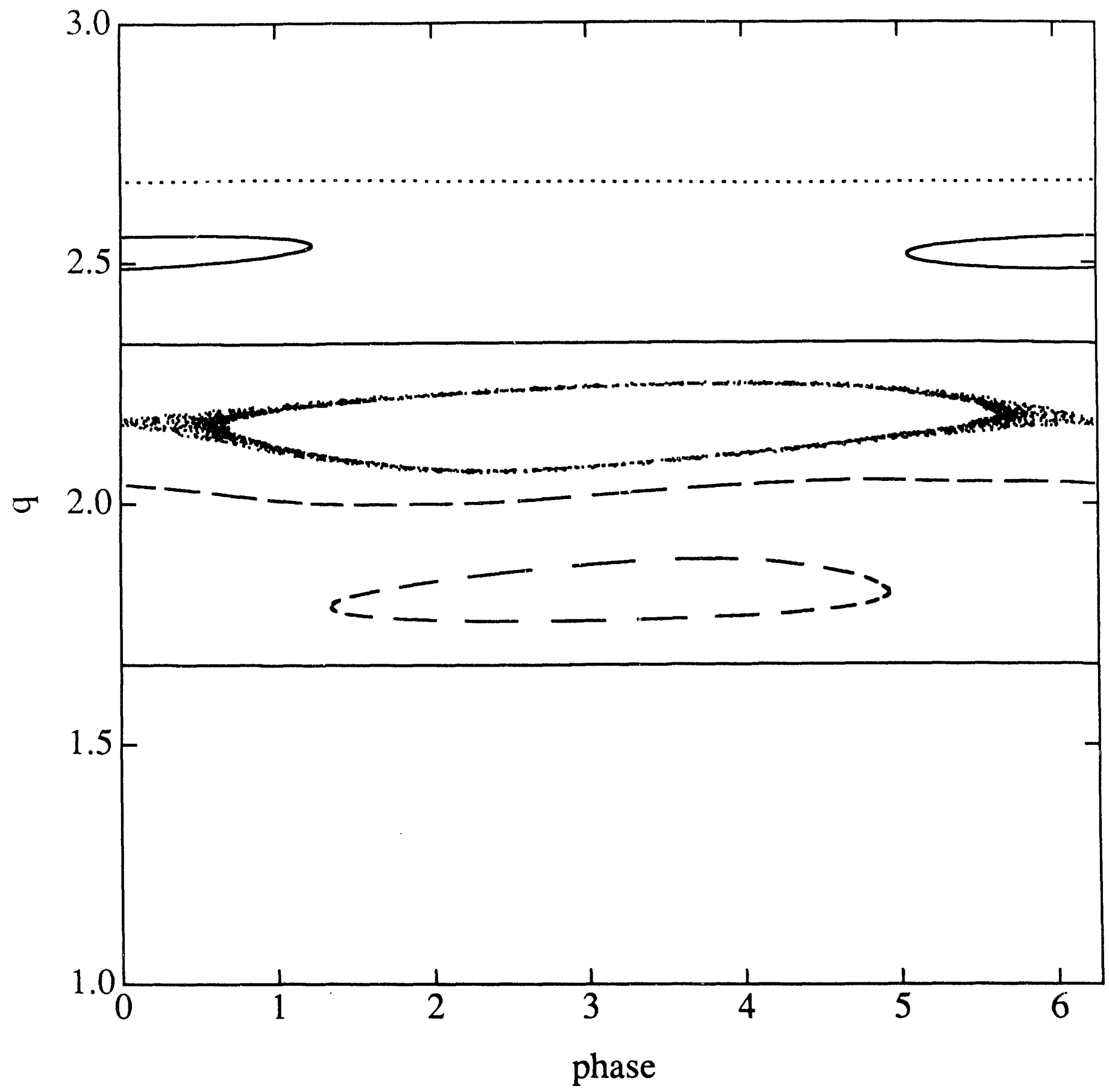

figure 1 


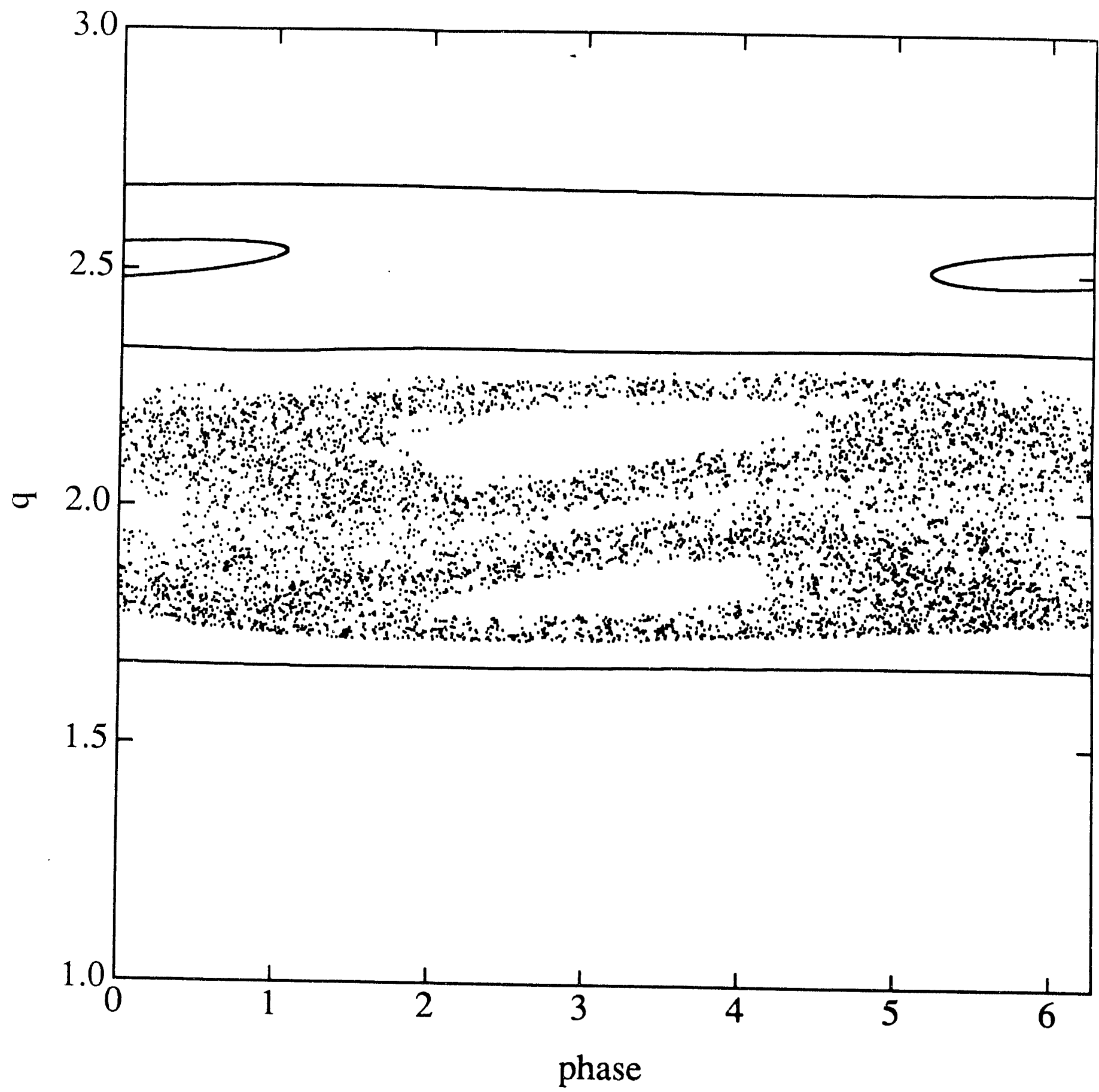

figure 2 


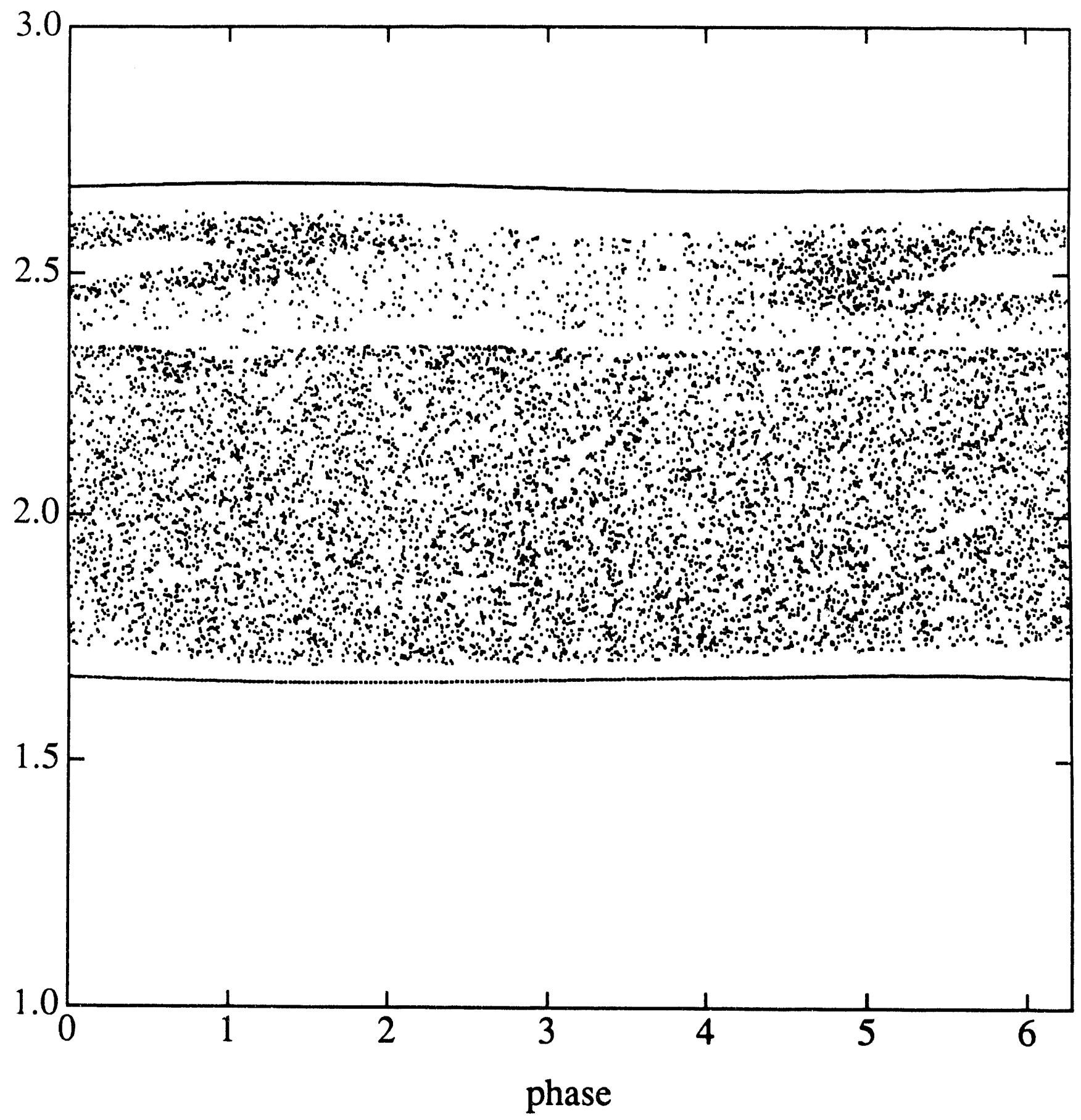

figure 3 


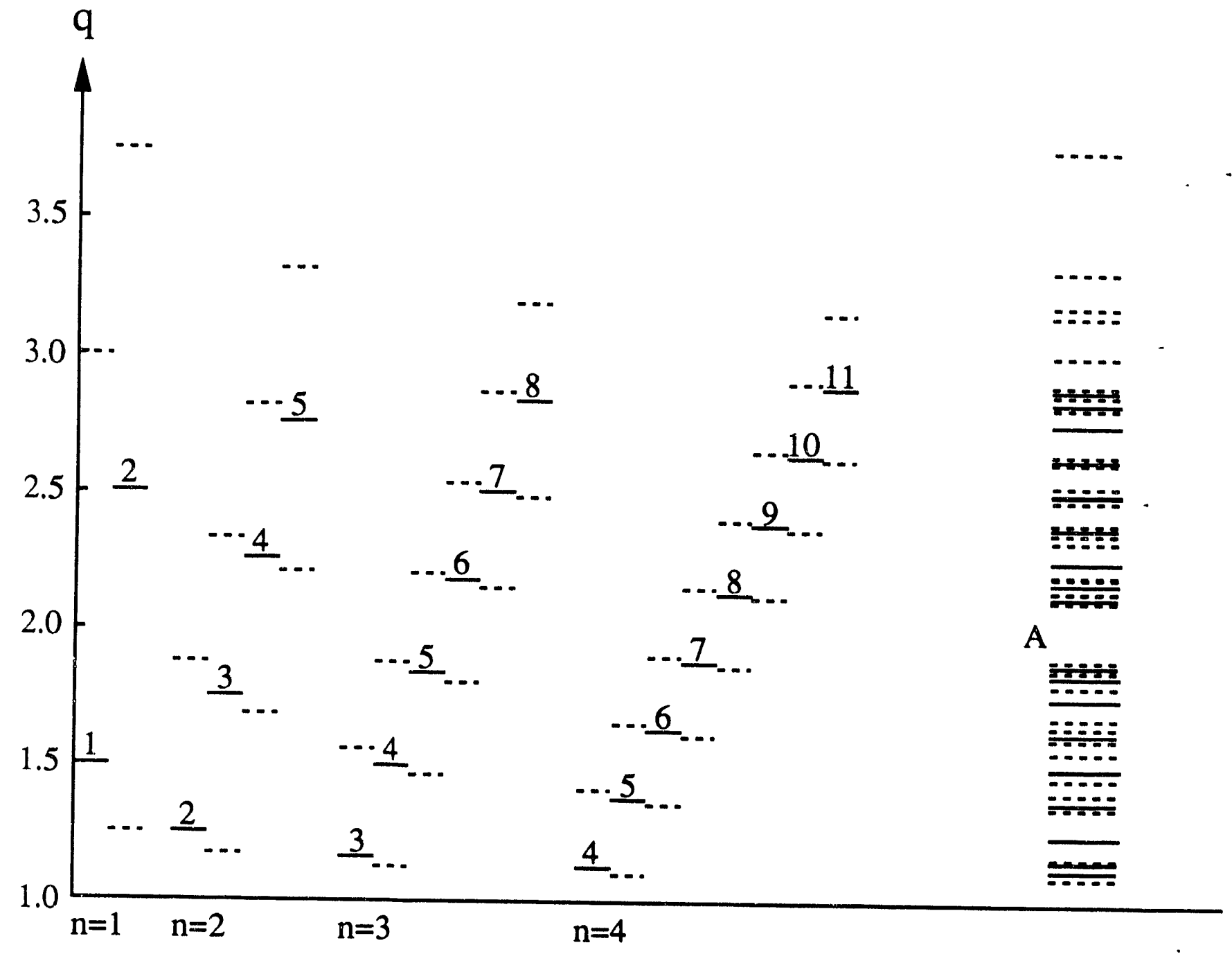




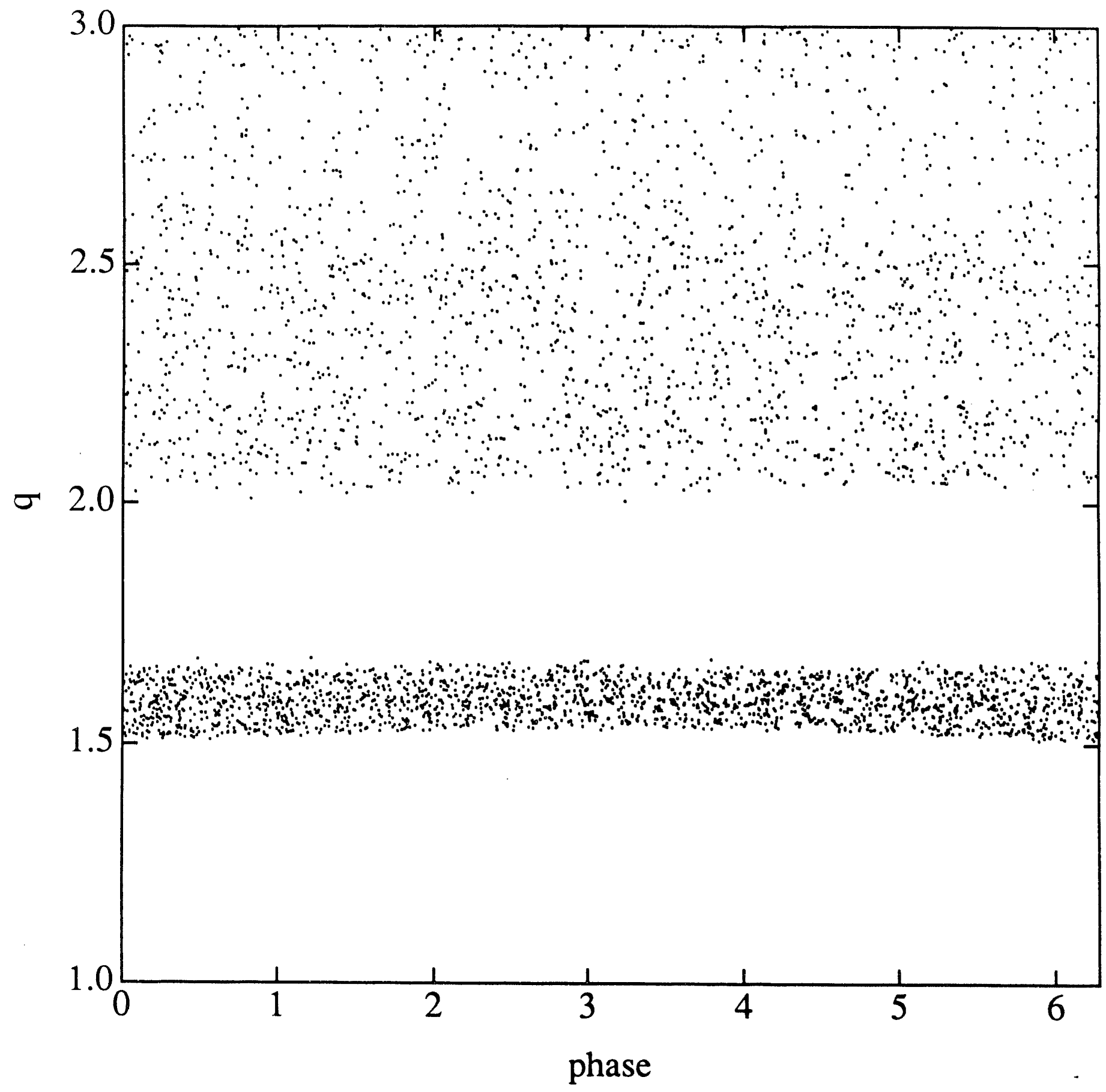

figure 5 


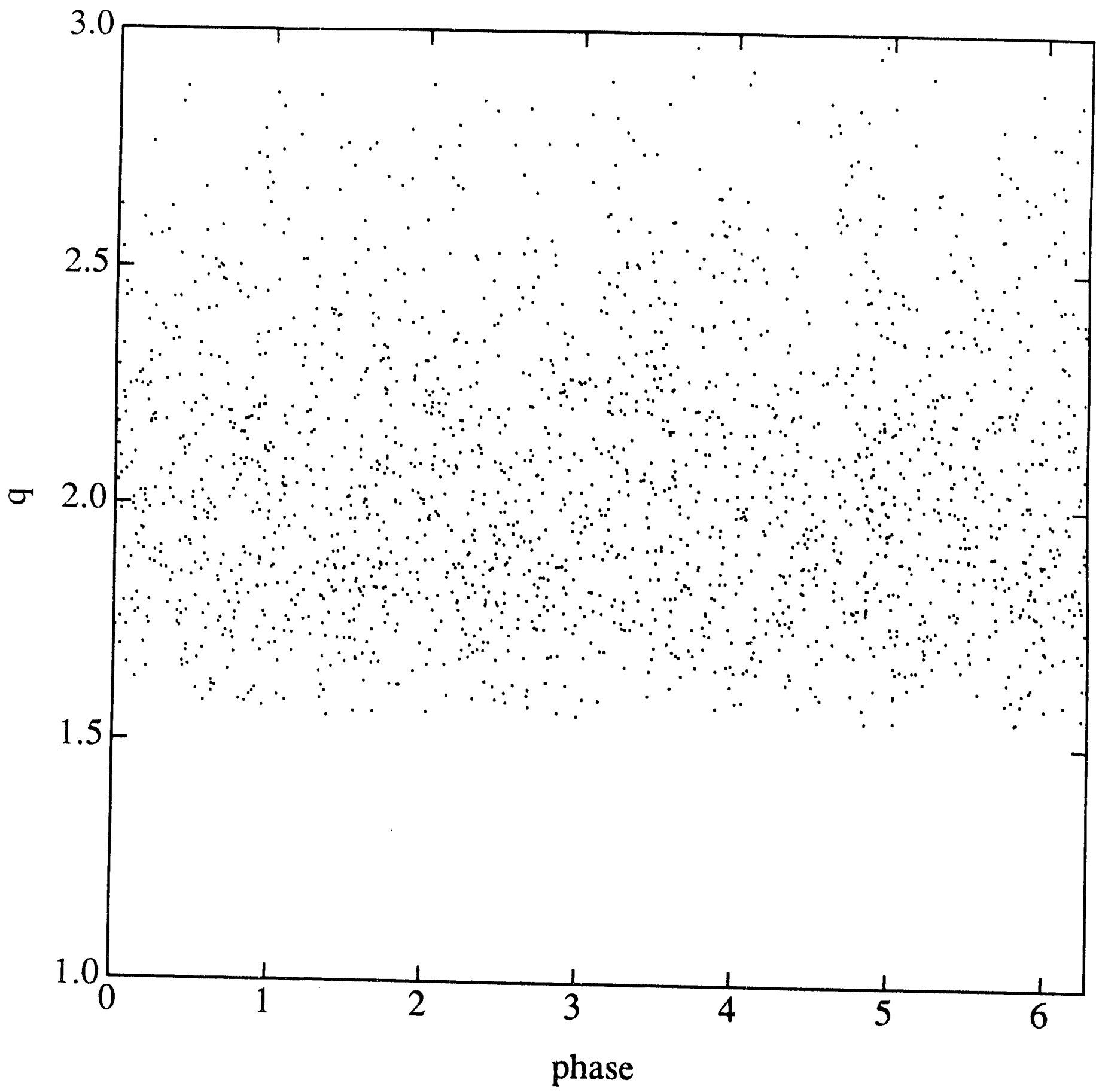

figure 6 

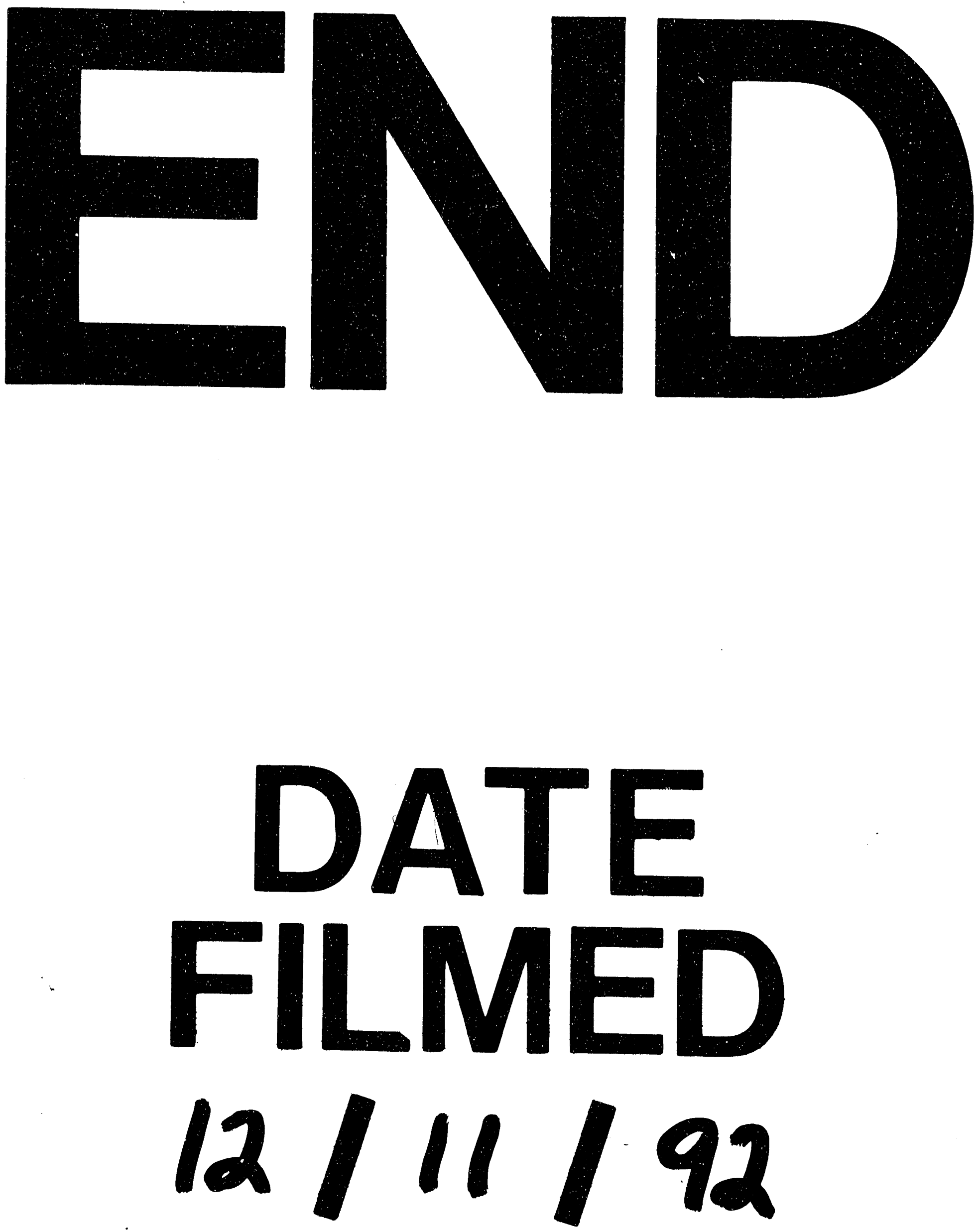


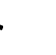

